\title{
Response to "Ethnic-Specific In Vitro-In Vivo Extrapolation and Physiologically Based Pharmacokinetic Approaches to Predict Cytochrome P450-Mediated Pharmacokinetics in Chinese Population: Opportunities and Challenges"
}

\author{
Zoe E. Barter • Geoffrey T. Tucker • \\ Karen Rowland-Yeo
}

Published online: 20 November 2013

(C) Springer International Publishing Switzerland 2013

We thank Dr Li and his colleagues for their comments, and agree that the development of population physiologically based pharmacokinetic models is always a work in progress pending the availability of additional data. The extra data that $\mathrm{Li}$ et al. [1] document relate to liver weight, cardiac output and cytochrome P450 (CYP) phenotype frequencies in Chinese subjects.

The additional data on liver weight for 1,114 subjects obtained at autopsy suggest a $9 \%$ higher mean value than the value from our meta-analysis of data from 342 subjects measured by computed tomography. Nevertheless, both values are lower than the mean value observed from our meta-analysis of Caucasian data.

In contrast to our analysis, which found a lower mean cardiac output in Chinese than in Caucasians, the data of Cao [2] cited by Dr Li and colleagues suggest the reverse. This difference should primarily affect the accuracy of the prediction of systemic clearance after intravenous administration of drugs with relatively high hepatic extraction ratios. Our predictions for plasma concentrations of midazolam, which has a hepatic extraction ratio of about 0.5 after intravenous injection, were similar to the observed data.

With the exception of the frequency of CYP2D6 ultrarapid metabolisers in Chinese (18\%), the CYP frequencies established from additional data by $\mathrm{Li}$ et al. [1] were similar to those that we reported. A review of the study of

Z. E. Barter $(\bowtie) \cdot$ G. T. Tucker · K. Rowland-Yeo

Simcyp Limited (a Certara company), Blades Enterprise Centre,

John Street, Sheffield S2 4SU, UK

e-mail: z.barter@simcyp.com

G. T. Tucker

Royal Hallamshire Hospital, University of Sheffield, Sheffield, UK
Guo et al. [3], quoted by Li et al. [1], indicates that their assessment was apparently not based on data for gene duplication but simply on the frequency of CYP2D $6^{*} 10$ alleles. Thus, the value of $18 \%$ is markedly higher than the figure of $1-2 \%$ documented for Asian populations in general $[4,5]$ and by our meta-analysis [6].

\section{References}

1. Li G, Yu G, Liu H, Zheng Q. Ethnic-specific in vitro-in vivo extrapolation and physiologically based pharmacokinetic approaches to predict cytochrome P450-mediated pharmacokinetics in Chinese population: opportunities and challenges. Clin Pharmacokinet. 2013. doi:10.1007/s40262-013-0119-9.

2. Cao JW. A regional study on main heart function parameters of Han Chinese and Tibetans in Sichuan, China [Masters dissertation]. Beijing: Chinese Academy of Medical Sciences and Peking Union Medical College; 2009.

3. Guo T, Zuo JL, Xia DY, Jia LH, Xiao Y, Guo HL. Genetic polymorphism analysis of cytochrome P450 (CYP)3A4, CYP2C9, CYP2C19 and CYP2D6 in Chinese Han and Hui population. Zhongguo Lin Chuang Yao Li Xue Za Zhi. 2012;28(5):281-4.

4. Johansson I, Oscarson M, Yue QY, Bertilsson L, Sjoqvist F, Ingleman-Sundberg M. Genetic analysis of the Chinese cytochrome P4502D locus: characterization of variant CYP2D6 genes present in subjects with diminished capacity for debrisoquine hydroxylation. Mol Pharmacol. 1994;46(3):452-9.

5. Wang SL, Huang JD, Lai MD, Liu BH, Lai ML. Molecular basis of genetic variation in debrisoquin hydroxylation in Chinese subjects: polymorphism in RFLP and DNA sequence of CYP2D6. Clin Pharmacol Ther. 1993;53(4):410-8.

6. Barter ZE, Tucker GT, Rowland-Yeo K. Differences in cytochrome P450-mediated pharmacokinetics between Chinese and Caucasian populations predicted by mechanistic physiologically based pharmacokinetic modelling. Clin Pharmacokinet. 2013;52(12):1085-100 